\title{
Actinides: How well do we know their stellar production?
}

\author{
S. Goriely and M. Arnould
}

\author{
Institut d'Astronomie et d'Astrophysique, Université Libre de Bruxelles, 1050 Brussels, Belgium
}

Received 2 August 2001 / Accepted 27 September 2001

\begin{abstract}
The reliable evaluation of the $r$-process production of the actinides and careful estimates of the uncertainties affecting these predictions are key ingredients especially in nucleo-cosmochronology studies based on the analysis of very metal-poor stars or on the composition of meteorites. This type of information is also required in order to make the best possible use of future high precision data on the actinide composition of galactic cosmic rays, of the local interstellar medium, or of meteoritic grains of presumed circumstellar origin. This paper provides the practitioners in these various fields with the most detailed and careful analysis of the $r$-process actinide production available to-date. This study is based on a version of the multi-event canonical model of the $r$-process which discards the largely used waiting point approximation. It considers also different combinations of models for the calculation of nuclear masses, $\beta$-decay and fission rates. Two variants of the model used to predict nuclear reaction rates are adopted. In addition, the influence of the level of $\mathrm{Pb}$ and $\mathrm{Bi}$ production by the $r$-process on the estimated actinide production is evaluated by relying on the solar abundances of these two elements. In total, thirty-two different cases are presented, and are considered to give a fair picture of the level of reliability of the predictions of the actinide production, at least in the framework of a simple $r$-process model. This simplicity is imposed by our inability to identify the proper astrophysical sites for the $r$-process. As a guide to the practitioners, constraints on the actinide yield predictions and associated uncertainties are suggested on grounds of the measured abundances of $r$-nuclides, including Th and U, in the star CS 31082-001, and under the critical and questionable assumption of the "universality" of the $r$-process. We also define alternative constraints based on the nucleo-cosmochronological results derived from the present actinide content of meteorites. Both sets of constraints suffer from serious problems. The first set does not hold in the likely situation of the non-universality of the $r$-process. The definition of the second set is made difficult by the necessity of using intricate galactic evolution models in order to interpret the meteoritic data. Our calculations of the actinide production combined with future data on the galactic cosmic ray actinide composition should help confirming that galactic cosmic rays are not fresh supernova ejecta. They should also provide a tool to discriminate between two competing models for the cosmic ray acceleration, one calling for an isolated supernova exploding in the ordinary old interstellar medium, and one envisioning a superbubble instead.
\end{abstract}

Key words. nuclear reactions - nucleosynthesis - abundances

\section{Introduction}

Actinides enter astrophysics in different ways. First, the use of ${ }^{232} \mathrm{Th},{ }^{235} \mathrm{U}$ and ${ }^{238} \mathrm{U}$ to estimate astrophysical ages has a long history, a milestone of which is the much celebrated piece of work of Fowler \& Hoyle (1960). For long, the field of nucleo-cosmochronology that has emerged from this paper has been aiming at the determination of the age $T_{\text {nuc }}$ of the nuclides from abundances in the material making up the bulk of the solar system. If indeed the composition of this material witnesses the long history of the compositional evolution of the Galaxy prior to the isolation of the solar material, a reliable evaluation of $T_{\text {nuc }}$ (clearly a lower bound to the age of the Universe) requires (i) the identification of radionuclides with half-lives

Send offprint requests to: S. Goriely, e-mail: sgoriely@astro.ulb.ac.be commensurable with estimated reasonable galactic ages (i.e. $t_{1 / 2} \gtrsim 10^{9} \mathrm{y}$ ), (ii) the construction of nucleosynthesis models that are able to provide the isotopic or elemental yields for these radionuclides, (iii) high quality data for the meteoritic abundances of the relevant nuclides, and, last but not least, (iv) the build-up of models for the evolution of the abundances of these nuclides in the Galaxy, primarily in the solar neighbourhood. All these requirements clearly make the chronometric task especially demanding. While everybody would agree this far, there are different ways to look at the question. The use of a socalled "model-independent" approach can at best set limits on $T_{\text {nuc }}$ (Schramm \& Wasserburg 1970). On the other hand, the "exponential model" introduced by Fowler \& Hoyle (1960) has been advocated by many over the years. It takes the stand that, given the presumed complexity of the chemical evolution of the galactic disk, it is by far 
preferable to describe its nucleosynthetic history by a simple function with some adjustable parameters. In contrast, it is considered by some that it is really worth studying nucleo-cosmochronology in the framework of models for the chemical evolution of the Galaxy in the solar neighbourhood which imperatively satisfy as many observational constraints as possible (e.g. Yokoi et al. 1983; Takahashi 1998).

The astrophysical importance of Th and $\mathrm{U}$ has been enhanced further with the observation of Th in some very metal-poor stars and of $U$ in one of them (Sneden et al. 1996; Cayrel et al. 2001). These measurements have raised the hope of a possible nuclear-based evaluation of the age of individual stars other than the Sun.

Other recent observational advances have triggered substantial interest in other actinides which are shorterlived than Th and U. This comes about following the measurement with unprecedented resolution of the Galactic Cosmic Ray (GCR) abundances of the $Z>70$ elements, including the actinides, using the Trek detector (Westphal et al. 1998). Further significant progress is expected in the determination of the GCR abundances of the actinides Th, U, Pu and $\mathrm{Cm}$ both with respect to each other and with respect to the Pt-group of elements. This is hoped to be achieved with the Extremely Heavy Cosmic Ray Composition Observer (ECCO), a detector similar to Trek currently under study for deployment on the International Space Station (Westphal et al. 2000). Precise abundance measurements of this type would yield an estimate of the time elapsed between the nucleosynthesis of the GCR actinides and their acceleration to GCR energies (the GCR actinide propagation time after acceleration is very short, i.e of the order of $2 \mathrm{My}$ ). Hence, they would help determining whether GCRs were accelerated out of fresh supernova ejecta, superbubble material, or old well-mixed galactic material.

Let us also mention the attempts to measure the local interstellar medium (ISM) ${ }^{244} \mathrm{Pu}$ content, which may have some interesting astrophysics implications. At present, this can be done through the analysis of dust grains of identified interstellar origin recovered in deep-sea sediments (e.g. Paul et al. 2001). In a near future, the determination of elemental and isotopic composition of the ISM grains will be a major goal of research with their recovery to Earth by the Stardust mission (Brownlee et al. 1996).

In all the fields referred to above, a necessary condition to interpret the observational data is to have at disposal $r$-process predictions for the production ratios at the sources of the actinides with half-lives typically in excess of about $10^{6} \mathrm{y}$, as well as ratios of these actinides to lower $Z$-element abundances. Most importantly, fair estimates of the uncertainties in these predicted abundances have also to be evaluated. Providing such a key information to the cosmo-chemistry, GCRs or interstellar dust practitioners is the main aim of this paper (Sect. 2). Attempts to derive constraints on the predictions of the actinide production from the solar system $r$-nuclide content and from abundance measurements in very metal-poor stars are discussed in Sects. 3 and 4. Brief considerations concerning the relations between our predictions and the GCR actinide content are presented in Sect. 5. Some conclusions are drawn in Sect. 6. At this point, we want to make clear that a detailed discussion of the impact of our nucleosynthesis predictions in the fields of nucleo-cosmochronology, GCR physics and interplanetary actinide content is largely out of the scope of this paper, and will possibly be the main concern of other works.

\section{Actinide production ratios}

\subsection{The multi-event canonical model of the r-process}

The $r$-process remains the most complicated nucleosynthetic process to model from the astrophysics as well as nuclear physics points of view (for a review see Arnould \& Takahashi 1999). On the nuclear physics side, the nuclear structure properties (such as the nuclear masses, deformation, ... ) of thousands of nuclei located between the valley of $\beta$-stability and the neutron drip line have to be known, as well as their interaction properties, i.e. the $(n, \gamma)$ and $(\gamma, n)$ rates, $\alpha$ - and $\beta$-decay half-lives and fission probabilities. Despite much recent experimental effort, those quantities for almost all the nuclei involved in the $r$-process remain unknown, so that they have to be extracted from theory, and are subject to unavoidable uncertainties. On top of these nuclear difficulties, the question of the astrophysical conditions under which the $r$-process can develop is far from being settled, all the proposed scenarios facing serious problems. For this reason, only parametric approaches, such as the so-called canonical model (Seeger et al. 1965) can be used to estimate the actinide production. The canonical model assumes that some stellar material composed solely of iron nuclei is subjected to neutron densities and temperatures that remain constant over the whole neutron irradiation time. Each event is therefore characterized by astrophysical conditions that are viewed as free parameters (temperatures $T$, neutron densities $N_{\mathrm{n}}$ and neutron irradiation times $t_{\text {irr }}$, which can be replaced by the related quantity $n_{\text {cap }}$, the average number of neutrons captured per iron seed). Their values are determined from a fit to the solar system composition of the abundances calculated for each canonical event (CEV).

In this paper, we use the multi-event model introduced by Bouquelle et al. (1996) (see also Goriely \& Arnould $1997)^{1}$. In view of our very poor knowledge of the precise astrophysical conditions of occurence of a given $r$-process, the suite of CEVs needed to approximate the yields of this process is clearly unknown as well. This is even more true

\footnotetext{
${ }^{1}$ As an unfortunate misconception persists in part of the $r$-process literature, we repeat here that the terminology "multi-event" does not refer necessarily to numerous stars (like supernovae) responsible for the production of $r$-nuclides, but rather to numerous CEVs characterized by different thermodynamic conditions. Such a suite of CEVs might for example well be associated to adjacent layers of a single supernova.
} 
if different types of $r$-process episodes have to be considered, at least if the assumption of the "universality" of the $r$-process yields is not adopted from the start (see Sect. 4). The multi-event model relies on an iterative inversion procedure in order to find the ensemble of CEVs which best fits a given abundance distribution, and in particular the solar system one, for a given nuclear input. Any change in this nuclear input translates in this approach into a different ensemble of CEVs in order to fit at best a given observed $r$-nuclide distribution. This is thus a unique and efficient tool, in particular to carry out a systematic study of the impact of nuclear uncertainties on the yield predictions (Goriely 1999). Some would argue that the multievent model has a real drawback because it can mask nuclear structure effects by introducing spurious CEVs. We would certainly concur with this criticism if indeed one would be able to distinguish at this point spurious CEVs from real ones. This ambiguity is made even more serious as our multi-event model as well as other more classical approaches (e.g. Cowan et al. 1999) make use of the CEV oversimplification. So far, we thus have to live with our inability to make a clear distinction between astrophysics and nuclear deficiencies of the $r$-process model (Goriely \& Arnould 1997).

The calculations referred to as "standard" in the following (see Table 1) are performed with the adoption of CEVs characterized by the astrophysical conditions (hereafter called SET1) $1.3 \leq T_{9} \leq 1.7\left(T_{9}\right.$ is the temperature in $\left.10^{9} \mathrm{~K}\right)$, and $10^{22} \leq N_{\mathrm{n}}\left[\mathrm{cm}^{-3}\right] \leq 10^{29}$. The CEVs are evaluated for the usual $10 \leq n_{\text {cap }} \leq 200$ range needed to produce elements between ${ }^{56} \mathrm{Fe}$ and the actinides (only CEVs with reasonable timescales $t_{\text {irr }}<2 \mathrm{~s}$ are considered). Note that the yields from each of the considered CEVs are not calculated under the waiting point approximation (in contrast to Goriely \& Clerbaux 1999). In these non-equilibrium conditions, the solution of a full reaction network is made necessary. When not available experimentally, the nuclear properties (in particular masses) are taken from the HFBCS-1 model of Goriely et al. (2001), the neutron capture and photodisintegration rates from Hauser-Feshbach calculations based on the HFBCS predictions (Goriely 2000), and from the gross theory (GT2) of Tachibana et al. (1990) for $\beta^{-}$decays and $\beta$-delayed neutron emissions. In addition, the spontaneous and $\beta$-delayed fission channels are included at times $t>t_{\text {irr }}$ only. The various fission probabilities (as well as $\alpha$ decays) are calculated according to the approximate prescriptions of Kodoma \& Takahashi (1975) with the experimental or ETFSI fission barriers (Mamdouh et al. 2001). In contrast, neutrino interactions are neglected. It is indeed meaningless to introduce them in a calculation which does not rely on a detailed astrophysical site.

When fitting a given observationally-based $r$-abundance distribution, the multi-event procedure is the same as the one described by Bouquelle et al. (1996), except that each nuclide is now given a weight inversely proportional to the uncertainty that is considered to affect its $r$-abundance. In the case of the solar system,

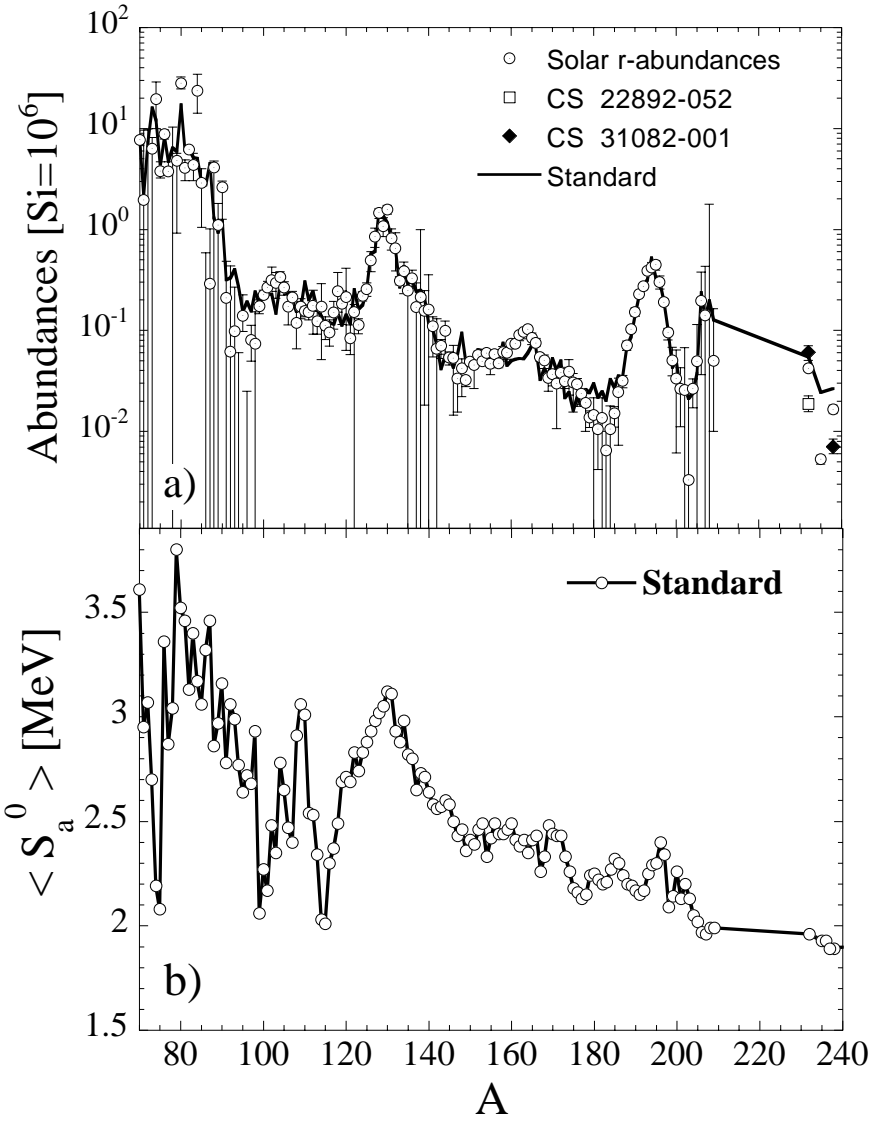

Fig. 1. a) Fit to the set of solar system $r$-abundances SOL1 derived from the standard calculations defined in the main text. The open square corresponds to the Th abundance observed in CS 22892-052 (Sneden et al. 1996), and the full diamonds to Th and U in CS31082-001 (Cayrel et al. 2001); b) Average values of $S_{\mathrm{a}}^{0}$ leading to the fit shown in a).

its $r$-nuclide content and associated uncertainties are discussed in detail by Goriely (1999). The data he derives define the solar system $r$-abundance set referred to in the following as "SOL1" (see Table 1). The multi-event fit to SOL1 obtained with the use of the standard calculations referred to above is displayed in Fig. 1. It involves a superposition of CEVs each of which being responsible for the production of a limited ensemble of $r$-nuclides. They can be characterized by the classically used quantity $S_{\mathrm{a}}^{0}(\mathrm{MeV})=\left(34.075-\log N_{\mathrm{n}}+\frac{3}{2} \log T_{9}\right) T_{9} / 5.04 . \mathrm{SET} 1$ includes CEVs for which $1.4 \lesssim S_{\mathrm{a}}^{0}(\mathrm{MeV}) \lesssim 4.2$. It is seen that a distribution of $S_{\mathrm{a}}^{0}$ decreasing roughly from 3.5 to $2 \mathrm{MeV}$ with increasing $A$-values is needed in order to reproduce the solar system distribution of $r$-nuclides. Case 1 of Table 1 provides the production of the actinides of astrophysical interest resulting from the fit of Fig. 1.

\subsection{How uncertain are the actinide abundance predictions?}

The predicted production of the actinides is obviously sensitive to the many, and still more or less uncertain, astrophysics and nuclear physics aspects of the $r$-process 
modelling. For all fields of space sciences where actinides enter, and in particular for cosmochronological purposes and for GCR astrophysics, it is of fundamental importance to know to what extent these uncertainties transpire into the calculated actinide synthesis. In fact, the problem of their production is particularly acute. There is no stable nuclide heavier than ${ }^{209} \mathrm{Bi}$, so that the actinide production can only be constrained by the stable nuclides lying some 30 mass units below. Moreover, in the simple $r$-process model considered here, the CEVs responsible for the actinide production do not contribute to the synthesis of the elements lighter than $\mathrm{Pb}$, including the third Os-Ir-Pt $r$-peak (Goriely \& Clerbaux 1999). Consequently, the estimated actinide production can be constrained solely by a fit to the $\mathrm{Pb}$ and $\mathrm{Bi}$ abundances.

We evaluate the uncertainties attached to the synthesis of the actinides by focussing on the solar system $r$-nuclide content. The conclusions derived from such a study can be generalized to other cases, and in particular to some very metal-poor stars, at least if the hypothesis of "universality" of the $r$-process holds (Sect. 4). In this analysis of the reliability of the actinide production, the multi-CEV model is adopted throughout. In other words, we put under the rug all the uncertainties of purely astrophysical nature affecting the $r$-process. This procedure finds its justification in the fact that these astrophysical uncertainties are almost impossible to quantify today, as the precise site(s) where the $r$-process develops is(are) largely unknown. Sticking to the multi-CEV model, we can, however, provide a limited evaluation of the astrophysical uncertainties by adopting a second set of conditions for the CEVs: $T_{9}=1.35$ and $10^{21} \leq N_{\mathrm{n}}\left[\mathrm{cm}^{-3}\right] \leq 10^{28}$. This set, referred to as SET2, is made of a much smaller number of CEVs than SET1, their $S_{\mathrm{a}}^{0}$ values ranging from about 1.7 to $3.5 \mathrm{MeV}$.

A second potential source of uncertainties relates to the fact that the relative $s$ - and $r$-contributions to the solar $\mathrm{Pb}$ and $\mathrm{Bi}$ remain obscure. This increases the difficulty to extrapolate abundance predictions reliably to the actinides. To estimate the uncertainty in the solar $\mathrm{Pb}$ and Bi $r$-abundances, we consider not only the set SOL1 defined above, but also the set referred to as SOL2 derived by Käppeler et al. (1989) from the classical $s$-process. The major difference between SOL1 and SOL2 lies in the predicted $r$-process contribution to the solar system ${ }^{208} \mathrm{~Pb}$ abundance: SOL2 and SOL1 predict values of 0.24 and in the (0 to 1.78) range, respectively (these $r$-abundances are expressed in the $\mathrm{Si}=10^{6}$ scale). The SOL1 predictions are so uncertain that the ${ }^{208} \mathrm{~Pb}$ abundance cannot provide any meaningful constraint in the $\mathrm{CEV}$ fitting procedure.

Another important source of uncertainties in the actinide abundance predictions is of nuclear physics nature. In order to evaluate its extent, we consider modifications to the various pieces of our standard nuclear input that are known to affect drastically the $r$-process predictions (Goriely \& Clerbaux 1999). These concern the nuclear masses, neutron capture and $\beta$-decay rates, as well as the probabilities of the fission channels that may open at one point or another during the $r$-process. With respect to the standard input, we replace

- the inclusion of fission just at times $t>t_{\text {irr }}$ by the due consideration of the fission processes during the neutron irradiation as well. This modification is called "Fission" in Table 1. In such conditions, the neutroninduced fission channel opens up on top of those already considered in the standard case;

- the HFBCS-1 nuclear masses by the following models: (i) HFB-1 (Samyn et al. 2001), (ii) ETFSI2 (Goriely 2000), (iii) ETFSI-Q (Pearson et al. 1996) which takes into account in a purely phenomenological way the still-debated strong quenching of the shell effect found in some microscopic calculations of highly neutron-rich nuclei, (iv) FRDM (Möller et al. 1995), or (v) the Duflo \& Zuker (1995; DZ) prescription based on a very different approach than the previously cited models, and which has proven its remarkable ability to predict experimentally known masses;

- the GT2 $\beta$-decay rates by (i) the FRDM+QRPA predictions of Möller et al. (1997), or (ii) the ETFSI+cQRPA estimates of Borzov \& Goriely (2000);

- the Hauser-Feshbach estimates of the neutron capture rates by the direct plus compound model $(\mathrm{CN}+\mathrm{DC})$ of Goriely (1998) which takes into account the possible existence of a low-lying E1 pygmy resonance and the contribution of direct captures of relevance for exotic neutron-rich nuclei.

Many studies have compared the quality of these nuclear models on grounds of available experimental data, as well as the differences in their predictions far away from the valley of $\beta$-stability. Their impact on the $r$-process nucleosynthesis has also been analyzed in various papers (e.g. Goriely 1998, 2001; Borzov \& Goriely 2000). Here, we restrict ourselves to an analysis of their predictions regarding the actinide synthesis.

Table 1 compares the abundances of $\mathrm{Pb}$ and of the actinides with half-lives $t_{1 / 2} \gtrsim 10^{6}$ y predicted by the multi-event calculations for the CEV SET1 and SET2 and for the solar $r$-abundances from SOL1 and SOL2. These different choices are mingled with the standard nuclear physics input defined in Sect. 2.1, or with various other combinations of nuclear models defined above. In all cases, the derived fits to the solar system content of $r$ nuclides up to Bi are of more or less equal quality (Goriely \& Clerbaux 1999). The corresponding abundance ${ }^{232} \mathrm{Th}_{\mathrm{f}}$ of ${ }^{232} \mathrm{Th}$ after decay of its shorter-lived progenitors ${ }^{236} \mathrm{U}$ and ${ }^{244} \mathrm{Pu}\left({ }^{232} \mathrm{Th}_{\mathrm{f}}={ }^{232} \mathrm{Th}+{ }^{236} \mathrm{U}+{ }^{244} \mathrm{Pu}\right)$, as well as the ratios $R_{232,238}={ }^{232} \mathrm{Th}_{\mathrm{f}} /{ }^{238} \mathrm{U}$ and $R_{235,238}=\left({ }^{235} \mathrm{U}+\right.$ $\left.{ }^{247} \mathrm{Cm}\right) /{ }^{238} \mathrm{U}$ are displayed in Table 2 . The considered large variety of nuclear models goes without any doubt along with large ranges of actinide predicted abundances. 
Table 1. Abundances (normalized to $\mathrm{Si}=10^{6}$ ) of $\mathrm{Pb}$ and of the actinides with half-lives $t_{1 / 2}>10^{6}$ y predicted by multi-event calculations with the astrophysical CEV sets SET1 or SET2 and solar system $r$-abundances SOL1 or SOL2. The calculations are also based on the various indicated combinations of nuclear inputs concerning masses, fission, $\beta$-decay and reaction rates (see text for more details). For each nuclide, the minimum and maximum abundances are underlined. The last three lines correspond to the recommended abundances with an estimate of the minimum and maximum values based on a selection of the above calculations, as described in Sect. 4. The selected cases are marked in bold in the first column.

\begin{tabular}{|c|c|c|c|c|c|c|c|c|c|c|c|}
\hline Case & SET & SOL & Nuclear & $\mathrm{Pb}$ & ${ }^{232} \mathrm{Th}$ & ${ }^{235} \mathrm{U}$ & ${ }^{236} \mathrm{U}$ & ${ }^{238} \mathrm{U}$ & ${ }^{237} \mathrm{~Np}$ & ${ }^{244} \mathrm{Pu}$ & ${ }^{247} \mathrm{Cm}$ \\
\hline 1 & 1 & 1 & Standard & $5.66 \mathrm{E}-01$ & $2.47 \mathrm{E}-02$ & $2.19 \mathrm{E}-02$ & $2.27 \mathrm{E}-02$ & $2.67 \mathrm{E}-02$ & $1.84 \mathrm{E}-02$ & $7.28 \mathrm{E}-03$ & $2.47 \mathrm{E}-03$ \\
\hline 2 & 1 & 1 & Fission & $5.75 \mathrm{E}-01$ & $2.53 \mathrm{E}-02$ & $2.26 \mathrm{E}-02$ & $2.35 \mathrm{E}-02$ & $2.44 \mathrm{E}-02$ & $1.90 \mathrm{E}-02$ & $7.43 \mathrm{E}-03$ & $2.57 \mathrm{E}-03$ \\
\hline 3 & 1 & 1 & HFB-1 & $82 \mathrm{E}-01$ & $2.16 \mathrm{E}-02$ & $2.21 \mathrm{E}-02$ & $2.11 \mathrm{E}-02$ & $2.57 \mathrm{E}-02$ & $1.56 \mathrm{E}-02$ & $1.01 \mathrm{E}-02$ & $2.39 \mathrm{E}-03$ \\
\hline 4 & 1 & 1 & ETFSI2 & $5.09 \mathrm{E}-01$ & $3.11 \mathrm{E}-02$ & $2.86 \mathrm{E}-02$ & $2.96 \mathrm{E}-02$ & $3.52 \mathrm{E}-02$ & $2.45 \mathrm{E}-02$ & 8.67E-03 & $4.58 \mathrm{E}-03$ \\
\hline 5 & 1 & 1 & ETFSI-Q & 6.07E-01 & $3.05 \mathrm{E}-02$ & $2.82 \mathrm{E}-02$ & $3.31 \mathrm{E}-02$ & $2.46 \mathrm{E}-02$ & $1.49 \mathrm{E}-02$ & $1.89 \mathrm{E}-02$ & $5.84 \mathrm{E}-03$ \\
\hline 6 & 1 & 1 & FRDM & $8.08 \mathrm{E}-01$ & $2.95 \mathrm{E}-02$ & $1.04 \mathrm{E}-01$ & $1.00 \mathrm{E}-01$ & $1.77 \mathrm{E}-01$ & $1.03 \mathrm{E}-01$ & $1.46 \mathrm{E}-01$ & $3.62 \mathrm{E}-02$ \\
\hline 7 & 1 & 1 & DZ & $4.65 \mathrm{E}-01$ & $\underline{8.62 \mathrm{E}-03}$ & 8.99E-03 & $9.20 \mathrm{E}-03$ & $1.13 \mathrm{E}-02$ & 7.73E-03 & $5.10 \mathrm{E}-03$ & $1.37 \mathrm{E}-03$ \\
\hline 8 & 1 & 1 & QRPA & $7.74 \mathrm{E}-01$ & $2.77 \mathrm{E}-02$ & $4.15 \mathrm{E}-02$ & $2.94 \mathrm{E}-02$ & $5.66 \mathrm{E}-02$ & $4.63 \mathrm{E}-02$ & $5.01 \mathrm{E}-02$ & $1.09 \mathrm{E}-02$ \\
\hline 9 & 1 & 1 & cQRPA & 7.69E-01 & $2.76 \mathrm{E}-02$ & 4.13E-02 & $2.92 \mathrm{E}-02$ & $5.61 \mathrm{E}-02$ & $4.59 \mathrm{E}-02$ & $4.97 \mathrm{E}-02$ & $1.08 \mathrm{E}-02$ \\
\hline 10 & 1 & 1 & $\mathrm{CN}+\mathrm{DC}$ & $6.53 \mathrm{E}-01$ & $.11 \mathrm{E}-02$ & $3.50 \mathrm{E}-02$ & 4.17E-02 & $3.67 \mathrm{E}-02$ & $2.58 \mathrm{E}-02$ & $3.26 \mathrm{E}-03$ & $3.48 \mathrm{E}-03$ \\
\hline 11 & 1 & 2 & HFBCS-1 & 7.93E-01 & $4.05 \mathrm{E}-02$ & $5.18 \mathrm{E}-02$ & $5.27 \mathrm{E}-02$ & $9.07 \mathrm{E}-02$ & $6.09 \mathrm{E}-02$ & $2.60 \mathrm{E}-02$ & $1.40 \mathrm{E}-02$ \\
\hline 12 & 1 & 2 & HFB-1 & 7.99E-01 & $3.50 \mathrm{E}-02$ & $3.75 \mathrm{E}-02$ & $3.42 \mathrm{E}-02$ & $5.20 \mathrm{E}-02$ & $3.29 \mathrm{E}-02$ & $2.58 \mathrm{E}-02$ & $4.36 \mathrm{E}-03$ \\
\hline 13 & 1 & 2 & ETFSI2 & $7.76 \mathrm{E}-01$ & $5.19 \mathrm{E}-02$ & $5.36 \mathrm{E}-02$ & $4.94 \mathrm{E}-02$ & $5.81 \mathrm{E}-02$ & $3.97 \mathrm{E}-02$ & $1.79 \mathrm{E}-02$ & $1.06 \mathrm{E}-02$ \\
\hline 14 & 1 & 2 & FRDM & $8.28 \mathrm{E}-01$ & $2.89 \mathrm{E}-02$ & $1.32 \mathrm{E}-01$ & $1.26 \mathrm{E}-01$ & $\underline{2.45 \mathrm{E}-01}$ & $\underline{1.37 \mathrm{E}-01}$ & $\underline{2.28 \mathrm{E}-01}$ & $\underline{5.72 \mathrm{E}-02}$ \\
\hline 15 & 1 & 2 & $\mathrm{DZ}$ & $7.53 \mathrm{E}-01$ & $2.75 \mathrm{E}-02$ & $4.51 \mathrm{E}-02$ & $4.48 \mathrm{E}-02$ & $1.00 \mathrm{E}-01$ & $6.01 \mathrm{E}-02$ & $6.52 \mathrm{E}-02$ & $2.20 \mathrm{E}-02$ \\
\hline 16 & 1 & 2 & $\mathrm{~N}+\mathrm{DC}$ & $8.07 \mathrm{E}-01$ & $6.25 \mathrm{E}-02$ & $5.39 \mathrm{E}-02$ & $6.49 \mathrm{E}-02$ & $6.85 \mathrm{E}-02$ & $4.72 \mathrm{E}-02$ & $6.29 \mathrm{E}-03$ & $6.85 \mathrm{E}-03$ \\
\hline 17 & 2 & 1 & Standard & $6.74 \mathrm{E}-01$ & $2.04 \mathrm{E}-02$ & $1.80 \mathrm{E}-02$ & $2.08 \mathrm{E}-02$ & $1.76 \mathrm{E}-02$ & $1.25 \mathrm{E}-02$ & $3.50 \mathrm{E}-03$ & $1.60 \mathrm{E}-03$ \\
\hline 18 & 2 & 1 & Fission & $6.60 \mathrm{E}-01$ & $1.96 \mathrm{E}-02$ & $1.71 \mathrm{E}-02$ & $1.97 \mathrm{E}-02$ & $1.51 \mathrm{E}-02$ & $1.18 \mathrm{E}-02$ & $3.32 \mathrm{E}-03$ & $1.49 \mathrm{E}-03$ \\
\hline 19 & 2 & 1 & $\mathrm{HFE}$ & $6.92 \mathrm{E}-01$ & $1.61 \mathrm{E}-02$ & $1.66 \mathrm{E}-02$ & $1.39 \mathrm{E}-02$ & $1.51 \mathrm{E}-02$ & $9.07 \mathrm{E}-03$ & 6.83E-03 & $1.22 \mathrm{E}-03$ \\
\hline 20 & 2 & 1 & ETFSI2 & $5.25 \mathrm{E}-01$ & $3.24 \mathrm{E}-02$ & $3.09 \mathrm{E}-02$ & $3.61 \mathrm{E}-02$ & $4.10 \mathrm{E}-02$ & $2.88 \mathrm{E}-02$ & $7.00 \mathrm{E}-03$ & $6.33 \mathrm{E}-03$ \\
\hline 21 & 2 & 1 & ETFSI-Q & $5.49 \mathrm{E}-01$ & $2.66 \mathrm{E}-02$ & $2.44 \mathrm{E}-02$ & $2.99 \mathrm{E}-02$ & $2.71 \mathrm{E}-02$ & $1.46 \mathrm{E}-02$ & $1.84 \mathrm{E}-02$ & $6.78 \mathrm{E}-03$ \\
\hline 22 & 2 & 1 & FRDM & $6.41 \mathrm{E}-01$ & $6.77 \mathrm{E}-02$ & $1.13 \mathrm{E}-01$ & $9.60 \mathrm{E}-02$ & $1.20 \mathrm{E}-01$ & 8.59E-02 & 8.71E-02 & $2.30 \mathrm{E}-03$ \\
\hline 23 & 2 & 1 & DZ & $4.94 \mathrm{E}-01$ & $1.27 \mathrm{E}-02$ & 7.95E-03 & $\underline{6.86 \mathrm{E}-03}$ & $\underline{9.42 \mathrm{E}-03}$ & $\underline{5.82 \mathrm{E}-03}$ & $4.22 \mathrm{E}-03$ & $\underline{1.01 \mathrm{E}-03}$ \\
\hline 24 & 2 & 1 & QRPA & $\underline{8.69 \mathrm{E}-01}$ & $2.61 \mathrm{E}-02$ & $3.47 \mathrm{E}-02$ & $2.87 \mathrm{E}-02$ & $8.05 \mathrm{E}-02$ & 7.07E-02 & $7.51 \mathrm{E}-02$ & $1.55 \mathrm{E}-02$ \\
\hline 25 & 2 & 1 & cQRPA & $\underline{4.11 \mathrm{E}-01}$ & $2.42 \mathrm{E}-02$ & 4.91E-02 & $4.68 \mathrm{E}-02$ & $8.92 \mathrm{E}-02$ & $5.09 \mathrm{E}-02$ & $2.02 \mathrm{E}-02$ & $1.64 \mathrm{E}-02$ \\
\hline 26 & 2 & 1 & $\mathrm{CN}+\mathrm{DC}$ & $5.65 \mathrm{E}-01$ & $2.87 \mathrm{E}-02$ & $2.87 \mathrm{E}-02$ & $3.66 \mathrm{E}-02$ & $2.32 \mathrm{E}-02$ & $1.85 \mathrm{E}-02$ & $\underline{3.19 \mathrm{E}-03}$ & $2.00 \mathrm{E}-03$ \\
\hline 27 & 2 & 2 & HFBCS-1 & 7.70E-01 & $2.99 \mathrm{E}-02$ & $3.87 \mathrm{E}-02$ & $4.54 \mathrm{E}-02$ & $5.55 \mathrm{E}-02$ & $3.55 \mathrm{E}-02$ & $1.07 \mathrm{E}-02$ & $9.28 \mathrm{E}-03$ \\
\hline 28 & 2 & 2 & HFB-1 & $7.75 \mathrm{E}-01$ & $2.72 \mathrm{E}-02$ & $2.42 \mathrm{E}-02$ & $2.15 \mathrm{E}-02$ & $2.88 \mathrm{E}-02$ & $1.72 \mathrm{E}-02$ & $1.57 \mathrm{E}-02$ & $3.47 \mathrm{E}-03$ \\
\hline 29 & 2 & 2 & ETFSI2 & 7.88E-01 & $5.90 \mathrm{E}-02$ & $6.10 \mathrm{E}-02$ & 7.07E-02 & $8.58 \mathrm{E}-02$ & $5.94 \mathrm{E}-02$ & $1.51 \mathrm{E}-02$ & $1.31 \mathrm{E}-02$ \\
\hline 30 & 2 & 2 & FRDM & $7.56 \mathrm{E}-01$ & $\underline{9.05 \mathrm{E}-02}$ & $\underline{1.68 \mathrm{E}-01}$ & $\underline{1.41 \mathrm{E}-01}$ & $1.80 \mathrm{E}-01$ & $1.30 \mathrm{E}-01$ & $1.37 \mathrm{E}-01$ & $2.78 \mathrm{E}-03$ \\
\hline 31 & 2 & 2 & DZ & 7.33E-01 & $4.88 \mathrm{E}-02$ & $5.07 \mathrm{E}-02$ & $4.13 \mathrm{E}-02$ & $9.14 \mathrm{E}-02$ & $5.56 \mathrm{E}-02$ & $6.30 \mathrm{E}-02$ & $1.81 \mathrm{E}-02$ \\
\hline 32 & 2 & 2 & $\mathrm{CN}+\mathrm{DC}$ & $8.14 \mathrm{E}-01$ & 4.83E-02 & 7.24E-02 & $9.79 \mathrm{E}-02$ & $5.54 \mathrm{E}-02$ & $4.64 \mathrm{E}-02$ & $3.95 \mathrm{E}-03$ & $6.09 \mathrm{E}-03$ \\
\hline Rec & 1 & 1 & Fission & $5.75 \mathrm{E}-01$ & $2.53 \mathrm{E}-02$ & $2.26 \mathrm{E}-02$ & $2.35 \mathrm{E}-02$ & $2.44 \mathrm{E}-02$ & $1.90 \mathrm{E}-02$ & $7.43 \mathrm{E}-03$ & $2.57 \mathrm{E}-03$ \\
\hline Min & & & & $5.09 \mathrm{E}-01$ & $2.53 \mathrm{E}-02$ & $2.26 \mathrm{E}-02$ & $2.15 \mathrm{E}-02$ & $2.32 \mathrm{E}-02$ & $1.46 \mathrm{E}-02$ & $3.19 \mathrm{E}-03$ & $2.00 \mathrm{E}-03$ \\
\hline Max & & & & 8.69E-01 & $6.77 \mathrm{E}-02$ & $1.13 \mathrm{E}-01$ & $1.00 \mathrm{E}-01$ & $1.77 \mathrm{E}-01$ & $1.03 \mathrm{E}-01$ & $1.46 \mathrm{E}-01$ & $3.62 \mathrm{E}-02$ \\
\hline
\end{tabular}

\section{Can the solar system $r$-nuclide content constrain the range of predicted actinide abundances?}

The long-lived ${ }^{232} \mathrm{Th}-{ }^{238} \mathrm{U}$ and ${ }^{235} \mathrm{U}-{ }^{238} \mathrm{U}$ pairs have been classically used to estimate the age of the $r$-nuclides (assumed to be roughly equal to the age of the Galaxy) from the present meteoritic content of these nuclides. The opinion has been expressed at several occasions that these pairs have just limited chronometric virtues (e.g. Yokoi et al. 1983; Arnould \& Goriely 2001). This opinion does not relate only to the uncertainties in the production ratios exemplified in Table 2, which could be increased still further if the oversimplification coming from the considered CEVs was removed. An additional source of worry comes from the still large uncertainties affecting the meteoritic Th and U abundances, which amount to at least $25 \%$ and $8 \%$, respectively (Grevesse et al. 1996). Last but not least, further problems arise because of the necessity of introducing the solar system-based nucleo-cosmochronology in chemical evolution models of the Galaxy. These models have to satisfy in the best possible way as many astronomical observables as possible. In addition, their internal consistency has to be checked by comparing the deduced actinide abundance ratios at the time of formation of the solar system with those adopted at the nucleosynthetic 
source. In fact, this consistency requirement is far from being trivial to fulfil. From the construction of a galactic evolution model generalized in order to include chronometric pairs, Yokoi et al. (1983) conclude first that the predicted $\left({ }^{235} \mathrm{U} /{ }^{238} \mathrm{U}\right)_{0}$ and $\left({ }^{232} \mathrm{Th} /{ }^{238} \mathrm{U}\right)_{0}$ ratios at the time $T_{\odot}$ of isolation of the solar system material from the galactic one about $4.6 \mathrm{~Gy}$ ago is only very weakly dependent on galactic ages, at least in the explored range from about 11 to $15 \mathrm{~Gy}$. This results largely from the expected rather weak time dependence of the stellar birthrate (except possibly at early galactic epochs, but a reliable information on these times is largely erased by the subsequent long period of chemical evolution). In this situation, the ${ }^{232} \mathrm{Th}-{ }^{238} \mathrm{U}$ and ${ }^{235} \mathrm{U}-238 \mathrm{U}$ pairs are unable to provide chronometric information which cannot be revealed by other methods. At best, they can provide results in agreement with conclusions derived from other techniques. As Yokoi et al. (1983) show, this is true at least if the $r$-process production ratios lie in the approximate ranges $1<{ }^{235} \mathrm{U} /{ }^{238} \mathrm{U}<$ 1.5 and $1.5<{ }^{232} \mathrm{Th}_{\mathrm{f}} /{ }^{238} \mathrm{U}<2$. If this is not the case, the adopted galactic evolution model simply does not provide any chronometric solution in the explored 11 to 15 Gy age range. As seen in Table 2, these constraints cannot be satisfied by any of the considered cases. The situation would not be as desperate if the lower and upper limits of the production ranges $R_{232,238}$ and $R_{235,238}$ were stretched by a value of 0.1 . Considering the intricacies of the galactic model adopted by Yokoi et al. (1983) to derive their chronological results, this small extension certainly does not hurt unsupportably their results. With such a procedure, we would be left with cases $1,3,4,7,8,9,13,20$, 22 and 30 of Tables 1 and 2.

\section{Can the observation of very metal-poor stars constrain the range of predicted actinide abundances?}

One might also confront the predicted range of actinide productions reported in Tables 1 and 2 with the observations of $r$-nuclides in old metal-poor stars. Compared with the case of the solar system discussed in Sect. 3, this chronometry has the advantage of allowing the economy of a galactic evolution model. Even so, the exercise of finding good reasons to reject some of the cases considered in Tables 1 and 2 is more risky than it might appear at first to some. The major origin of the difficulties lies in the necessity to make the assumption that the $r$-process is "universal". In other words, the observed patterns of $r$-nuclide abundances in metal-poor stars have to be considered as exactly solar. This is indeed the only way to take the largest possible advantage of the observed metalpoor star content of $\mathrm{Th}$ and $\mathrm{U}$ by bringing them to the status of chronometers.

Before embarking on the problem of deriving constraints, let us first briefly discuss the validity of the universality hypothesis. In contrast to a widely spread opinion, Goriely \& Arnould (1997) (see also Goriely \& Clerbaux 1999; Arnould \& Goriely 2001) consider that the observed convergence of the solar and of the metalpoor stars CS 22892-052 and HD115444 abundance patterns in the $56 \leq Z \leq 76$ range does in no way demonstrate the universality of the r-process, without excluding it, however. This conclusion fully applies as well to the recent abundance determinations of $50 \leq Z \leq 70$ elements in 22 metal-poor $r$-process-rich stars (Johnson \& Bolte 2001). In addition, there has been some bad news for the many proponents of the $r$-process universality with the observations by Cayrel et al. (2001) of the very metal-poor $r$-process-enriched halo star CS 31082-001. While a large similarity between the $56<Z<70$ element patterns in CS 22892-052, HD115444 and CS 31082-001 is reported, the abundances in the latter star differ significantly from those of the other two stars in the $Z>70$ range, including Th. In these conditions, the universality assumption would lead to quite odd chronometric conclusions (Arnould \& Goriely 2001). In particular, the Th/Eu ratio in CS 31082-001 is about 3.2 times larger than in CS 22892-052. Hence, under the universality assumption, CS 22892-052 (with $[\mathrm{Fe} / \mathrm{H}]=-3.1$ ) predates CS 31082001 (with $[\mathrm{Fe} / \mathrm{H}]=-2.9$ ) by $24 \mathrm{~Gy}$, and would thus be about 36 Gy old.

The $\mathrm{Pb} / \mathrm{Th}$ ratios observed in CS 22892-052 $(\log \epsilon(\mathrm{Pb} / \mathrm{Th})=1.80 \pm 0.40)$ and in CS 31082-001 $(\log \epsilon(\mathrm{Pb} / \mathrm{Th})<0.76)$ may run down as well the universality hypothesis. A correlation indeed exists between the $r$-process production of $\mathrm{Pb}$ and $\mathrm{Th}$, the abundances of these two elements increasing or decreasing concomitantly (see Fig. 1 of Goriely \& Clerbaux 1999). In these conditions, and if the universality of the $\mathrm{Pb} / \mathrm{Th}$ ratio is assumed, the observed $\mathrm{Pb} / \mathrm{Th}$ values turn out to be discrepant by a factor of about 10, at least if the two stars have roughly the same age. If this is indeed the case (which is not a farfetched assumption in view of their similar $[\mathrm{Fe} / \mathrm{H}]$ ratio), either the universality assumption is invalid, and a specific actinide-producing $r$-process has to be called for, or the $\mathrm{Pb}$ in CS 22892-052 is largely of $s$-process origin. This could well be the case if a $\mathrm{Pb}$ pollution by a low-metallicity AGB star could be invoked (in particular if CS 22892-052 would be a binary star). As shown by Goriely \& Siess (2001) and Van Eck et al. (2001), the $s$-process in extremely low-metallicity AGB stars indeed leads almost exclusively to the production of $\mathrm{Pb}$, in such a way that none of the elements lighter than $\mathrm{Pb}$ would see its abundance affected by the $s$-process. It would be of substantial interest to find ways to discriminate between the $r$-process non-universality and the $s$-process pollution scenarios. Even if the assumption of a universal $r$-process appears to be more and more fragile with time, we dare suppose in the following that it indeed holds in order to examine if constraints can be put in such a favourable situation on the nuclear and astrophysical models for use in $r$-process calculations, and consequently on the actinide production.

To make things clear, the universality assumption means nothing more and nothing less than the following: all possible combinations of CEVs found in nature 
Table 2. Same as Table 1 for total ${ }^{232} \mathrm{Th}_{\mathrm{f}}={ }^{232} \mathrm{Th}+{ }^{236} \mathrm{U}+{ }^{244} \mathrm{Pu}, R_{232,238}={ }^{232} \mathrm{Th}_{\mathrm{f}} /{ }^{238} \mathrm{U}, R_{235}, 238=\left({ }^{235} \mathrm{U}+{ }^{247} \mathrm{Cm}\right) /{ }^{238} \mathrm{U}$, and ages $T_{\mathrm{U}, \mathrm{Th}}^{*}$ and $T_{\mathrm{U}, \text { Eu }}^{*}$ (in Gy) of CS 31082-001 based on the U/Th and U/Eu cosmochronometries. The minimum and maximum values for each entry are underlined. Recommended ranges of values of these quantities are also provided (see Sect. 4 for details). As in Table 1, the selected cases are marked in bold in the first column.

\begin{tabular}{|c|c|c|c|c|c|c|c|c|}
\hline Case & SET & SOL & Nuclear & ${ }^{232} \mathrm{Th}_{\mathrm{f}}$ & $R_{232,238}$ & $R_{235,238}$ & $T_{\mathrm{U}, \mathrm{Th}}^{*}$ & $T_{\mathrm{U}, \mathrm{Eu}}^{*}$ \\
\hline 1 & 1 & 1 & Standard & 0.0547 & 2.05 & 0.91 & 13.55 & 8.38 \\
\hline 2 & 1 & 1 & Fission & 0.0561 & 2.30 & 1.03 & 12.48 & 7.81 \\
\hline 3 & 1 & 1 & HFB-1 & 0.0528 & 2.05 & 0.95 & 13.54 & 8.14 \\
\hline 4 & 1 & 1 & ETFSI2 & 0.0694 & 1.97 & 0.94 & 13.92 & 10.16 \\
\hline 5 & 1 & 1 & ETFSI-Q & 0.0826 & 3.36 & 1.39 & 8.94 & 7.86 \\
\hline 6 & 1 & 1 & FRDM & 0.2760 & 1.56 & 0.79 & 16.14 & 20.52 \\
\hline 7 & 1 & 1 & DZ & $\underline{0.0229}$ & 2.03 & 0.92 & 13.66 & 2.88 \\
\hline 8 & 1 & 1 & QRPA & $\overline{0.1072}$ & 1.89 & 0.93 & 14.30 & 13.20 \\
\hline 9 & 1 & 1 & cQRPA & 0.1064 & 1.90 & 0.93 & 14.30 & 13.15 \\
\hline 10 & 1 & 1 & $\mathrm{CN}+\mathrm{DC}$ & 0.0860 & 2.34 & 1.05 & 12.31 & 10.43 \\
\hline 11 & 1 & 2 & HFBCS-1 & 0.1192 & 1.31 & 0.73 & 17.73 & 16.23 \\
\hline 12 & 1 & 2 & HFB-1 & 0.0950 & 1.83 & 0.80 & 14.65 & 12.67 \\
\hline 13 & 1 & 2 & ETFSI2 & 0.1192 & 2.05 & 1.10 & 13.56 & 13.38 \\
\hline 14 & 1 & 2 & FRDM & $\underline{0.3832}$ & 1.56 & 0.77 & 16.11 & $\underline{22.60}$ \\
\hline 15 & 1 & 2 & DZ & 0.1376 & 1.37 & 0.67 & 17.31 & 16.86 \\
\hline 16 & 1 & 2 & $\mathrm{CN}+\mathrm{DC}$ & 0.1337 & 1.95 & 0.89 & 14.02 & 14.43 \\
\hline 17 & 2 & 1 & Standard & 0.0447 & 2.53 & 1.11 & 11.58 & 5.73 \\
\hline 18 & 2 & 1 & Fission & 0.0426 & 2.82 & 1.23 & 10.57 & 4.74 \\
\hline 19 & 2 & 1 & HFB-1 & 0.0368 & 2.43 & 1.18 & 11.97 & 4.75 \\
\hline 20 & 2 & 1 & ETFSI2 & 0.0754 & 1.84 & 0.91 & 14.57 & 11.14 \\
\hline 21 & 2 & 1 & ETFSI-Q & 0.0749 & 2.76 & 1.15 & 10.77 & 8.49 \\
\hline 22 & 2 & 1 & FRDM & 0.2508 & 2.09 & 0.96 & 13.39 & 18.03 \\
\hline 23 & 2 & 1 & DZ & 0.0238 & 2.53 & 0.95 & 11.59 & 1.71 \\
\hline 24 & 2 & 1 & QRPA & 0.1299 & 1.61 & $\underline{0.62}$ & 15.81 & 15.46 \\
\hline 25 & 2 & 1 & cQRPA & 0.0911 & 1.02 & 0.73 & 20.09 & 16.12 \\
\hline 26 & 2 & 1 & $\mathrm{CN}+\mathrm{DC}$ & 0.0685 & 2.96 & 1.33 & 10.13 & 7.47 \\
\hline 27 & 2 & 2 & HFBCS-1 & 0.0860 & 1.55 & 0.87 & 16.18 & 13.08 \\
\hline 28 & 2 & 2 & HFB-1 & 0.0644 & 2.24 & 0.96 & 12.74 & 8.87 \\
\hline 29 & 2 & 2 & ETFSI2 & 0.1448 & 1.69 & 0.86 & 15.38 & 15.87 \\
\hline 30 & 2 & 2 & FRDM & 0.3681 & 2.04 & 0.95 & 13.61 & 20.64 \\
\hline 31 & 2 & 2 & $\mathrm{DZ}$ & 0.1532 & 1.68 & 0.75 & 15.45 & 16.28 \\
\hline 32 & 2 & 2 & $\mathrm{CN}+\mathrm{DC}$ & 0.1501 & 2.71 & 1.42 & 10.94 & 13.06 \\
\hline $\operatorname{Rec}$ & 1 & 1 & Fission & 0.0561 & 2.30 & 1.03 & 12.48 & 7.81 \\
\hline Min & & & & 0.0561 & 1.31 & 0.62 & 8.94 & 7.47 \\
\hline Max & & & & 0.2760 & 3.36 & 1.42 & 17.73 & 20.52 \\
\hline
\end{tabular}

always result in the same final abundance pattern. It is important to acknowledge that the precise characteristics of each of the CEVs which may contribute to the universal mix are unknown, as well as the relative level of the contribution of each of the CEVs to the mix. In such conditions, it cannot be excluded that different combinations of different individual CEVs lead to the same final mix. An improper evaluation of this situation may lead to spurious constraints on the nuclear models to be used as input to the $\mathrm{CEV}$ calculations. Another source of spurious constraints might arise by focussing on the quality of a fit to a single nuclide, even in a quite crucial region, like the $\mathrm{Pb}$ one (e.g. Cowan et al. 1999). Such a highly punctual quality of fit may be quite misleading in the evaluation of the merits of a global nuclear input and of the associated predictions of the actinide production.

One might imagine gaining some constraints from Th alone. The ages of CS 22892-052 and CS 31082-001 derived from a confrontation between the observed Th abundances and those displayed in Table 1 vary in the $3 \lesssim T^{*}[\mathrm{~Gy}] \lesssim 60$ and $0 \lesssim T^{*}[\mathrm{~Gy}] \lesssim 37$ ranges, respectively. Obviously, some of these ages are meaningless when reference is made to other age determination techniques (reviewed in e.g. von Hippel et al. 2001). On such grounds, one might thus be tempted to eliminate right away some of the nuclear models used in Table 1. This stand is perfectly legitimate in the simplistic astrophysics scenario of superposed CEVs we have adopted (Sect. 2.1). In contrast, by so doing, one 
may face the danger of reaching wrong conclusions if more realistic $r$-process models were considered. The absolute Th production is indeed likely to be highly dependent on these models. The consequences of this situation are aggravated by the fact that the ages derived from Th are especially sensitive to its precise yield predictions, as a result of its long half-life $\left(t_{1 / 2} \approx 14 \mathrm{~Gy}\right)$.

Let us thus turn to safer ways to derive meaningful constraints. One can in particular rely on accurate measurements of U/Th in individual low-metallicity stars (Arnould \& Takahashi 1999; Goriely \& Clerbaux 1999), these two actinides being produced concomitantly. A $\mathrm{U} / \mathrm{Th}$ ratio has been reported for the star CS 31082-001 (Cayrel et al. 2001; Hill et al. 2001). This is real good news, even if the situation is not free of observational and theoretical difficulties. The former ones are discussed by Cayrel et al. (2001), and are vividly illustrated by the very recent re-evaluation of the ThII and UII line strengths by Nilsson et al. (2001, 2001a) leading to the revised values of $\log \varepsilon(\mathrm{U} / \mathrm{H})=-1.83$ and $\log \varepsilon(\mathrm{Th} / \mathrm{H})=-0.89$. The resulting $\log (\mathrm{U} / \mathrm{Th})$ for CS 31082-001 is $-0.94 \pm 0.09$ (Cayrel, private communication) instead of the originally derived value of -0.74 (Cayrel et al. 2001; Hill et al. 2001). On the theoretical side, uncertainties, if they cannot of course be eliminated, are largely reduced, however. The predicted stellar ages lie in the restricted $9 \lesssim T^{*}[\mathrm{~Gy}] \lesssim 18$ range (Table 2). None of the $r$-process calculations shown in Table 2 can be excluded for sure on the basis of the $\mathrm{U} / \mathrm{Th}$ chronometry. This situation just translates the enhanced reliability of the predictions of the $\mathrm{U} / \mathrm{Th}$ ratio compared to the ones based on a single actinide.

Some constraints on the actinide production could be gained from the development of chronometries based on other $r$-process pairs, ideally to be used in conjunction with $\mathrm{U} / \mathrm{Th}$. One of these pairs which has already been adopted quite often in the past is Th/Eu. This choice has of course been dictated by available observations, but is in fact quite unfortunate from a theoretical point of view. It indeed combines the drawback coming from the long Th lifetime, as stressed above, with the inconvenience that $\mathrm{Eu}$ and $\mathrm{Th}$ are not produced in the same CEV, so that the $\mathrm{Th} / \mathrm{Eu}$ production ratio is expected to be quite drastically model dependent. Considering now the U/Eu pair, it is, of course, expected to be as model dependent as Th/Eu. It has however the pleasing feature of involving $\mathrm{U}$, which has a lifetime about three times shorter than Th. This has the advantage of making chronometric conclusions less sensitive to the precise production of $\mathrm{U}$ than to the one of $\mathrm{Th}$. So, although it also requires the assumption of $r$-process universality, the U/Eu chronometry may be safer than the $\mathrm{Th} / \mathrm{Eu}$ one. Table 2 indicates that the use of U/Eu leads to a CS 31082-001 age in the $7 \lesssim T^{*}[\mathrm{~Gy}] \lesssim 23$ range. Under the constraint that the age of CS 31082-001 obtained from $\mathrm{U} / \mathrm{Th}\left(T_{\mathrm{U}, \mathrm{Th}}^{*}\right)$ and $\mathrm{U} / \mathrm{Eu}\left(T_{\mathrm{U}, \mathrm{Eu}}^{*}\right)$ should be the same, we reject the cases for which $T_{\mathrm{U}, \mathrm{Th}}^{*}$ and $T_{\mathrm{U}, \mathrm{Eu}}^{*}$ differ by more than 5 Gy. From the retained cases, we suggest in Tables 1 and 2 recommended, minimum and maximum values for each predicted abundance and age.
Note that U/Os would be a valuable chronometric pair as well, in principle at least. We do not use it, however. The star CS 31082-001 indeed exhibits a Os overabundance of about 0.35 dex with respect to the universal pattern (Hill et al. 2001). This clearly contradicts the universality assumption which is the basis of all the chronometric considerations making use of metal-poor stars. The measured Os overabundance would imply a CS 31082-001 age $T_{\mathrm{U}, \text { Os }}^{*}$ exceeding $T_{\mathrm{U}, \mathrm{Eu}}^{*}$ by about 5 Gy. More generally, this situation creates some discomfort about the whole cosmochronometric virtues of the actinides in metal-poor stars.

Some closing remarks are in order at this point. First, we want to restate that the reported constraints strictly relate to the simplistic $\mathrm{CEV}$ model of the $r$-process, and put totally under the rug uncertainties related to the very nature of the $r$-process and the likely intricate astrophysical conditions that prevail during its development. A detailed treatment of uncertainties of such a nature is largely out of reach in view of the poor characterization of the true astrophysical context in which an analysis of this type should have to be conducted. Second, the constraints adopted to select the recommended actinide productions and their ranges of variations given in Tables 1 and 2, while admitedly highly subjective, appear reasonable to the authors only under the assumption of the universality of the r-process. At discussed above, this basic assumption appears to be more and more questionable as data accumulate. As a direct consequence, the derived constraints are increasingly unsecure.

\section{The Galactic cosmic ray actinide composition}

As stressed in Sect. 1, measurements of actinide abundances relative to each other and to the Pt-group of elements is of very special interest in order to help determining whether GCRs are fresh supernova ejecta, or superbubble material, or old galactic material.

It is generally taken for granted today that supernova explosions are the most probable GCR energy source. It is believed that individual supernova remnants may be responsible for the acceleration of external, swept-up ISM, with at most a very tiny contribution of internal, nucleosynthetically processed material (Meyer \& Ellison 1999; Ellison \& Meyer 1999). Observation of GCR actinides could confirm this scenario. Massive star supernova explosions are not random in the Galaxy, however, and concentrate strongly in $\mathrm{OB}$ associations. In fact, fireworks of sequential explosions of tens of massive stars lasting for periods as short as a few million years could create "multiple supernova remnants". These can grow into so-called "superbubbles" made of hot tenuous plasma most commonly observed from their X-ray emission in our and nearby galaxies (e.g. Spitzer 1990). Superbubbles might well be privileged galactic locations for the acceleration of matter to GCR energies (Higdon et al. 1998; Parizot 2000, 2001; Bykov 2001). Just as in the case of isolated remnants, each superbubble remnant accelerates external, swept-up 


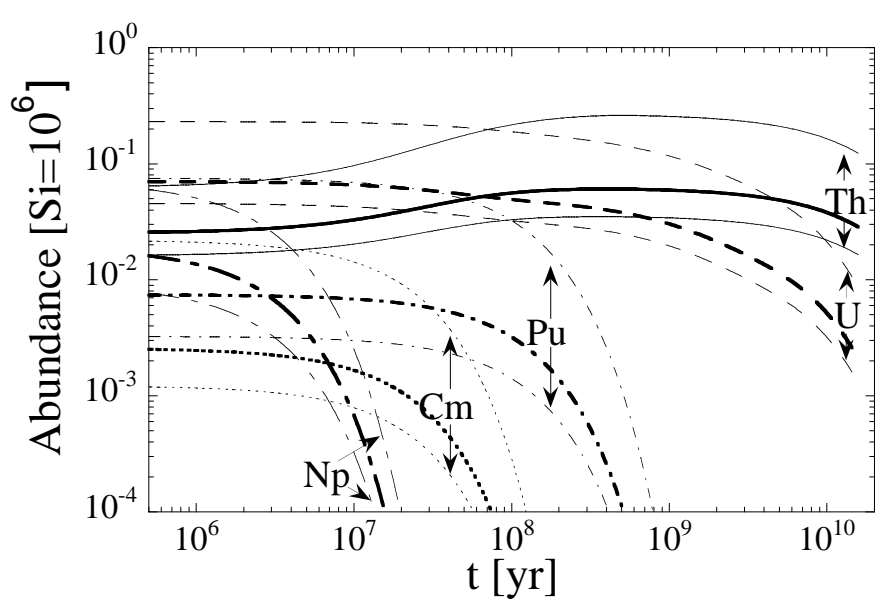

Fig. 2. Time variations of the elemental abundances of the actinides of interest for GCR studies. A single $r$-process production is assumed at time zero. The thick and thin lines correspond to the recommended values and their lower or upper limits given in Table 1.

superbubble material. This material, while predominantly ordinary ISM evaporated from nearby clouds, is, however, significantly contaminated by the recent ejecta of previous local supernovae. In addition, turbulent acceleration should take place steadily throughout the superbubble gas. So, more fresh supernova ejecta may be expected in GCRs from superbubbles than from isolated supernovae. This results from the study of both the superbubble dynamics and from considerations about the synthesis of the light elements Li, Be and B in the early galaxy (e.g. Parizot 2000, 2001). This increased fraction of fresh ejecta also nicely accounts for the GCR ${ }^{22} \mathrm{Ne}$ anomaly (Meynet et al. 2001).

An accurate measurement of the actinide GCR composition which is within the reach of the planned ECCO detector (Sect. 1) could provide an additional and specific way of distinguishing between the isolated supernova remnant and supperbubble scenarios for the source of the GCRs (Westphal et al. 2000). Indeed, the relatively short lifetimes of the superbubbles imply a young age (say $<30 \mathrm{My}$ ) for the emerging GCRs. Such an age constraint is relaxed in the case of isolated supernova remnants. So, as noted by Westphal et al. (2000), GCRs originating from supperbubbles would likely be young enough for containing a significant amount of ${ }^{244} \mathrm{Pu}$ and ${ }^{247} \mathrm{Cm}$ the lifetimes of which are commensurable with the superbubble ones. Concomitantly, the presence of ${ }^{247} \mathrm{Cm}$ in the GCRs and their implied young age would be the indication that $\mathrm{Th}$, $\mathrm{U}$, and $\mathrm{Pu}$ have abundance ratios close to their $r$-process yields. In such conditions, first hand constraints on the actinide production by the $r$-process could be gained, in addition to quality information on the origin and age of the GCRs.

We provide in Fig. 2 some predicted abundances of interest for the quantitative interpretation of the future GCR actinide measurements. We retain only the cases of Table 2 satisfying the constraints set in Sect. 4 from the spectroscopic analyses of very metal-poor stars. A detailed discussion of these results is out of the scope of this paper.

\section{Conclusion}

The reliable evaluation of the $r$-process production of the actinides and careful estimates of the uncertainties affecting these predictions are key ingredients especially in nucleo-cosmochronology studies based on the analysis of very metal-poor stars or on the composition of meteorites. This type of information is also required in order to prepare making the best possible use of future high precision data on the GCR actinide composition, to establish a more quantitative confrontation with an expected growing body of measurements of the actinide content of the interstellar medium in the solar neighbourhood, or even of meteoritic grains of suspected circumstellar origin.

This paper provides the practitioners in these various fields with the most detailed and careful analysis of the $r$-process actinide production available to-date. These predictions are based on a version of the multi-event canonical model of the $r$-process which discards the largely used waiting point approximation. This approach is especially well suited for evaluating uncertainties which emerge from the adopted nuclear physics. It is acknowledged that this model is astrophysically oversimplified. However, going far beyond this simplification is close to impossible in view of the present lack of knowledge about the site(s) of the $r$-process and of the physical conditions prevailing during its development. Thirty-two different combinations of models for the calculation of nuclear masses, $\beta$-decay rates, $\beta$-delayed probabilities, as well as fission processes are considered. Two variants of the model used to predict nuclear reaction rates are adopted. The impact of uncertainties in the $r$-process production of the closest $\mathrm{Pb}$ and Bi $r$-nuclides is also analyzed.

For the defenders of the universality of the $r$-process, we show how this property can help defining constraints on the actinide yield predictions and associated uncertainties. To derive these constraints, we take advantage of the simultaneous $\mathrm{Th}$ and $\mathrm{U}$ measurements in the star CS 31082-001 and we impose somewhat arbitrarily that the age derived from the observed $\mathrm{U} / \mathrm{Th}$ ratio should not differ from the $\mathrm{U} / \mathrm{Eu}$ age by more than 5 Gy. We acknowledge that other criteria could be defined on grounds of the metal-poor star actinide data and of the $r$-process universality. From the information provided by Tables 1 and 2, the readers may in fact set up their own choice of criteria and define their corresponding own constraints and recommended actinide productions, if they find them more appropriate. We stress most strongly that these constraints are just meaningless if the universality of the r-process does not hold, a situation that we clearly favour. In fact, the universality of the $r$-process appears to be less and less likely as data accumulate.

We also discuss briefly constraints that could be put on the $r$-process nuclear physics input from nucleocosmochronological results based on the confrontation 
between meteoritic actinide abundances and predictions relying on a model for the chemical evolution of the Galaxy in the solar neighbourhood. The problem of setting up this second set of constraints does not relate to the likely non-universality of the $r$-process, but instead to the intricacies introduced by the necessity to rely on models for the chemical evolution of the Galaxy. As a consequence, providing solid values for the actinide production, or even reliable "error bars" on this production, remains a highly risky exercise. Clearly, much is left for the future.

Finally, our calculations of the actinide production are shown to provide the necessary tools for using at best future data about the galactic cosmic ray actinide composition. The confrontation between these observations and our predictions should help confirming that galactic cosmic rays are not fresh supernova ejecta. They should also provide a way of discriminating between two competing models for their acceleration: the isolated supernova remnant exploding in ordinary, old ISM and the superbubble scenario.

Acknowledgements. We thank R. Cayrel for bringing to our attention the still unpublished re-evaluation of the Th and $\mathrm{U}$ abundances in the star CS 31082-001. We also thank J.-P. Meyer and A. J. Westphal for fruitful discussions concerning the actinide content of GCRs, and for their guidance in the field. J.-P. Meyer and R. Cayrel are also acknowledged for their careful reading of the manuscript and for suggestions which have helped improving the manuscript. S.G. is FNRS Research Associate.

\section{References}

Arnould, M., \& Takahashi, K. 1999, Rep. Prog. Phys., 62, 393 Arnould, M., \& Goriely, S. 2001, in Astrophysical Ages and Time Scales, ed. T. von Hippel, N. Manset, \& C. Simpson, ASP Conf. Ser., 252

Borzov, I., \& Goriely, S. 2000, Phys. Rev. C, 62, 035501

Bouquelle, V., Cerf, N., Arnould, M., Tachibana, T., \& Goriely, S. 1996, A\&A, 305, 1005

Brownlee, D. E., Burnett, D., Clark, B., et al. 1996, in Physics, Chemistry, and Dynamics of Interplanetary Dust, ed. B. A. S. Gustafson, \& M. S. Hanner, ASP Conf. Ser., 104,223

Bykov, A. M. 2001, Space Sci. Rev., in press

Cayrel, R., Hill, V., Beers, T. C., et al. 2001, Nature, 409, 691

Cowan, J. J., Pfeiffer, B., Kratz, K.-L., et al. 1999, ApJ, 521, 194

Duflo, J., \& Zuker, A. 1995, Phys. Rev., C52, R23

Ellison, D. C., \& Meyer, J. P. 1999, in LiBeB, Cosmic Rays, and Related X- and Gamma-Rays, ed. R. Ramaty, E. Vangioni-Flam, M. Cassé, \& K. Olive, ASP Conf. Ser., 171, 207

Fowler, W. A., \& Hoyle, F. 1960, Ann. Phys., 10, 280

Goriely, S. 1998, Phys. Lett. B, 436, 10

Goriely, S. 1999, A\&A, 342, 881

Goriely, S. 2000, in Tenth Int. Symp. on Capture Gamma-Ray Spectroscopy and Related Topics, ed S. Wender, AIP Conf. Proc., 529, 287

Goriely, S. 2001, Hyperfine Interactions, in press
Goriely, S., \& Arnould, M. 1997, A\&A, 322, L29

Goriely, S., \& Clerbaux, B. 1999, A\&A, 342, 881

Goriely, S., \& Siess, L. 2001, A\&A, 378, L25

Goriely, S., Tondeur, F., \& Pearson, J. M. 2001, At. Data Nucl. Data Tables, 77, 311

Grevesse, N., Noels, A., \& Sauval, A. J. 1996, in Cosmic Abundances, ed. S. S. Holt, \& G. Sonneborn, ASP Conf. Ser., 99, 117

Higdon, J. C., Lingenfelter, R. E., \& Ramaty, R. 1998, ApJ, 487, 182

Hill, V., Plez, B., Cayrel, R., \& Beers, T. C. 2001, in Astrophysical Ages and Time Scales, ed. T. von Hippel, N. Manset, \& C. Simpson, ASP Conf. Ser., in press

Johnson, J. A., \& Bolte, A. 2001, ApJ, 554, 888

Käppeler, F., Beer, H., \& Wisshak, K. 1989, Rep. Prog. Phys., 52,945

Kodoma, T., \& Takahashi, K. 1975, Nucl. Phys., A239, 489

Mamdouh, A., Pearson, J. M., Rayet, M., \& Tondeur, F. 2001, Nucl. Phys. A, 679, 337

Meyer, J. P., \& Ellison, D. C. 1999, in LiBeB, Cosmic Rays, and Related X- and Gamma-Rays, ed. R. Ramaty, E. Vangioni-Flam, M. Cassé, \& K. Olive, ASP Conf. Ser., 171, 187

Meynet, G., Arnould, M., Paulus, G., \& Maeder, A. 2001, Space Sci. Rev., in press

Möller, P., Nix, J. R., Myers, W. D., \& Swiatecki, W. J. 1995, At. Data Nucl. Data Tables, 59, 131

Möller, P., Nix, J. R., \& Kratz, K.-L. 1997, At. Data Nucl. Data Tables, 66, 185

Nilsson, H., Ivarsson, S., Johansson, S., \& Lundberg, H. 2001, A\&A, submitted

Nilsson, H., Zhang, Z., Lundberg, H., Johansson, S., \& Nordström, B. 2001a, A\&A, submitted

Parizot, E. 2000, A\&A, 362, 786

Parizot, E. 2001, Space Sci. Rev., in press

Paul, M., Valenta, A., Ahmad, I., et al. 2001, preprint [astro-ph/0106205]

Pearson, J. M., Nayak, R. C., \& Goriely, S. 1996, Phys. Lett. B, 387, 455

Samyn, M., Goriely, S., Pearson, J. M., Heenen, P.-H., \& Tondeur, F. 2001, Nucl. Phys. A, in press

Schramm, D. N., \& Wasserburg, G. J. 1970, ApJ, 162, 57

Seeger, P. A., Fowler, W. A., \& Clayton, D. D. 1965, ApJS, 11,121

Sneden, C., McWilliam, A., Preston, G. W., et al. 1996, ApJ, 467,819

Spitzer, L. Jr. 1990, ARA\&A, 28, 71

Tachibana, T., Yamada, M., \& Yoshida, Y. 1990, Progr. Theor. Phys., 84, 641

Takahashi, K. 1998, in Tours Symposium on Nuclear Physics III, ed. M. Arnould, M. Lewitowicz, Yu. Ts. Oganessian, et al., AIP Conf. Proc., 425, 616

Van Eck, S., Goriely, S., Jorissen, A., \& Plez, B. 2001, Nature, 412, 793

von Hippel, T., Manset, N., \& Simpson, C. (eds.) 2001, Astrophysical Ages and Time Scales, ASP Conf. Ser., in press

Westphal, A. J., Price, P. B., Weaver, B. A., \& Afanasiev, V. G. 1998, Nature, 396, 50

Westphal, A. J., Weaver, B. A., Solarz, M., Lin, C.-L., \& Zuñiga, L. 2000, in Proc. Int. Cosmic Ray Conf., ed. D. Kieda, et al., 160

Yokoi, K., Takahashi, K., \& Arnould, M. 1983, A\&A, 117, 65 